\title{
NECESSARY AND SUFFICIENT CONDITION FOR THE LAMPERTI INVARIANCE PRINCIPLE
}

UDC 519.21

\author{
ALFREDAS RAČKAUSKAS AND CHARLES SUQUET
}

\begin{abstract}
Let $\left(X_{i}\right)_{i \geq 1}$ be an i.i.d. sequence of random variables with null expectation and variance one, $S_{n}:=X_{1}+\cdots+X_{n}$, and $\xi_{n}$ the random polygonal line with vertices $\left(k / n, S_{k}\right), k=0,1, \ldots, n$. By a theorem of Lamperti (1962), if $X_{1}$ has a moment of order $p>2$, then $n^{-1 / 2} \xi_{n}$ weakly converges to the standard Brownian motion $W$ in the Hölder space $H_{\alpha}$ for $\alpha<1 / 2-1 / p$. We prove that a necessary and sufficient condition for the $H_{\alpha}$-weak convergence of this process to $W$ is that $\mathrm{P}\left(\left|X_{1}\right|>t\right)=o\left(t^{-p(\alpha)}\right)$, where $p(\alpha)=1 /(1 / 2-\alpha)$. As an illustration, we present an application to the change point detection under the epidemic alternative.
\end{abstract}

\section{INTRODUCTION}

Let $X_{1}, \ldots, X_{n}, \ldots$ be i.i.d. mean zero random variables. Set $S(0)=0$ and

$$
S(t)=\sum_{k \leq t} X_{k}, \quad t>0,
$$

and consider the process

$$
\xi_{n}(t)=S([n t])+(n t-[n t]) X_{[n t]+1}, \quad t \in[0,1] .
$$

The classical Donsker-Prokhorov invariance principle states that if $\mathrm{E} X_{1}^{2}=1$, then

$$
n^{-1 / 2} \xi_{n} \stackrel{\mathcal{D}}{\longrightarrow} W
$$

in $C[0,1]$, where $(W(t), t \in \mathbf{R})$ is a standard Wiener process and $\stackrel{\mathcal{D}}{\longrightarrow}$ denotes the convergence in distribution. Since (1) yields the central limit theorem, the finiteness of the second moment of $X_{1}$ therefore is necessary.

Lamperti (1962) considered the convergence (1) with respect to a stronger Hölderian topology. Let us recall his result. For $0<\alpha<1$, let $H_{\alpha}^{0}[0,1]$ be the set of real-valued continuous functions $x:[0,1] \rightarrow \mathbf{R}$ such that

$$
\lim _{\delta \rightarrow 0} \omega_{\alpha}(x, \delta)=0,
$$

where

$$
\omega_{\alpha}(x, \delta)=\sup _{\substack{t, s \in[0,1], 0<|t-s|<\delta}} \frac{|x(t)-x(s)|}{|s-t|^{\alpha}} .
$$

The set $H_{\alpha}^{0}[0,1]$ is a Banach space when endowed with the norm

$$
\|x\|_{\alpha}=|x(0)|+\omega_{\alpha}(x, 1) .
$$

2000 Mathematics Subject Classification. Primary 60F17; Secondary 60B12.

Research supported by a cooperation agreement CNRS/LITHUANIA (4714).

(C)2004 American Mathematical Society 
Lamperti (1962) proved that if $0<\alpha<\frac{1}{2}$ and $\mathrm{E}\left|X_{1}\right|^{p}<\infty$, where $p>1 /\left(\frac{1}{2}-\alpha\right)$, then (1) holds in the Hölder space $H_{\alpha}^{0}[0,1]$. This result was derived again by Kerkyacharian and Roynette (1991) by another method using analysis given by Ciesielski (1960) of Hölder spaces by triangular functions. Further generalizations were given by Erickson (1981), Hamadouche (1998), and Račkauskas and Suquet (1999).

Considering a symmetric random variable $X_{1}$ such that

$$
\mathrm{P}\left(X_{1} \geq u\right)=1 /\left(2 u^{p}\right), \quad u \geq 1,
$$

Lamperti (1962) noticed that the sequence $\left(n^{-1 / 2} \xi_{n}\right)$ is not tight in $H_{\alpha}^{0}[0,1]$ for

$$
\alpha=1 / 2-1 / p .
$$

It is then hopeless in general to look for an invariance principle in $H_{\alpha}^{0}[0,1]$ without some moment assumption beyond the square integrability of $X_{1}$.

The main result of this note is the following theorem whose proof is given in Section 3.

Theorem 1. Assume that $0<\alpha<\frac{1}{2}$ and $p=1 /\left(\frac{1}{2}-\alpha\right)$. Then

$$
n^{-1 / 2} \xi_{n} \stackrel{\mathcal{D}}{\longrightarrow} W \quad \text { in the space } H_{\alpha}^{0}[0,1]
$$

if and only if

$$
\lim _{t \rightarrow \infty} t^{p} \mathrm{P}\left(\left|X_{1}\right| \geq t\right)=0 .
$$

Corollary 2. Let $0<\alpha<\frac{1}{2}$ and $p=1 /\left(\frac{1}{2}-\alpha\right)$. Then for each $\delta>0$

$$
n^{-1 / 2+\alpha} \max _{0<|k-j|<\delta n} \frac{|S(k)-S(j)|}{|k-j|^{\alpha}} \stackrel{\mathcal{D}}{\longrightarrow} \omega_{\alpha}(W, \delta)
$$

if and only if condition (2) holds.

For statistical applications of results like Corollary 2, it is very desirable to compute the exact distribution of $\|W\|_{\alpha}$ and $\omega_{\alpha}(W, \delta)$. Unfortunately no explicit formula seems to be known for such distributions. To remedy this drawback, we propose in Section 4 to use the Ciesielski sequential equivalent norms, for which it is rather easy to give an explicit formula and to perform practical computations.

As an illustration, we show in Section 5 how both the classical and sequential Hölder norms can be used in the change point problem under the so-called epidemic alternative.

\section{Preliminaries}

First let us fix some notation for the Haar and Faber-Schauder functions. A simple way to parameterize these families is to use directly the dyadic numbers. Denote by $D_{j}$ the set of dyadic numbers in $[0,1]$ of level $j$, i.e.,

$$
D_{0}=\{0,1\}, \quad D_{j}=\left\{(2 l-1) 2^{-j} ; 1 \leq l \leq 2^{j-1}\right\}, \quad j \geq 1 .
$$

So the countable set $D$ of dyadic numbers of $[0,1]$ may be written as

$$
D=\bigcup_{j=0}^{\infty} D_{j} .
$$

For notational convenience, we put

$$
D^{*}:=D \backslash\{0\} .
$$

For $r \in D_{j}, j \geq 0$, write

$$
r^{-}:=r-2^{-j}, \quad r^{+}:=r+2^{-j} .
$$


For $r \in D_{j}, j \geq 1$, the $L^{2}[0,1]$ normalized Haar function $H_{r}$ is defined on $[0,1]$ by

$$
H_{r}(t)= \begin{cases}+\left(r^{+}-r^{-}\right)^{-1 / 2}=+2^{(j-1) / 2} & \text { if } t \in\left(r^{-}, r\right], \\ -\left(r^{+}-r^{-}\right)^{-1 / 2}=-2^{(j-1) / 2} & \text { if } t \in\left(r, r^{+}\right], \\ 0, & \text { otherwise. }\end{cases}
$$

In the special case $j=0$, as the support $\left[r^{-}, r^{+}\right]$would extend beyond $[0,1]$ we have to modify the above formula to keep the $L^{2}[0,1]$ normalization. This leads us to define

$$
H_{0}(t):=-\mathbb{1}_{[0,1]}(t), \quad H_{1}(t):=\mathbb{1}_{[0,1]}(t) .
$$

The sequence $\left\{H_{r} ; r \in D^{*}\right\}$ is an orthonormal basis of the Hilbert space $L^{2}[0,1]$.

For $r \in D_{j}, j \geq 1$, the triangular Faber-Schauder functions $\Lambda_{r}$ are continuous, piecewise affine with support $\left[r^{-}, r^{+}\right]$and taking the value 1 at $r$ :

$$
\Lambda_{r}(t)= \begin{cases}\left(t-r^{-}\right) /\left(r-r^{-}\right)=2^{j}\left(t-r^{-}\right) & \text {if } t \in\left(r^{-}, r\right], \\ \left(r^{+}-t\right) /\left(r^{+}-r\right)=2^{j}\left(r^{+}-t\right) & \text { if } t \in\left(r, r^{+}\right], \\ 0, & \text { otherwise. }\end{cases}
$$

In the special case $j=0$, we just take the restriction to $[0,1]$ in the above formula, so

$$
\Lambda_{0}(t)=1-t, \quad \Lambda_{1}(t)=t, \quad t \in[0,1] .
$$

The $\Lambda_{r}$ 's have the $C[0,1]$ normalization: $\sup _{0 \leq t \leq 1}\left|\Lambda_{r}(t)\right|=1$. They are linked to the $H_{r}$ 's in the general case $r \in D_{j}, j \geq 1$, by

$$
\Lambda_{r}(t)=2\left(r^{+}-r^{-}\right)^{-1 / 2} \int_{0}^{t} H_{r}(s) d s=2^{(j+1) / 2} \int_{0}^{t} H_{r}(s) d s
$$

and in the special case $j=0$ by

$$
\Lambda_{0}(t)=1+\int_{0}^{t} H_{0}(s) d s, \quad \Lambda_{1}(t)=\int_{0}^{t} H_{1}(s) d s .
$$

The sequence $\left\{\Lambda_{r} ; r \in D\right\}$ is a Schauder basis of $C[0,1]$ (see, e.g., [5]). Each $x \in C[0,1]$ has a unique expansion

$$
x=\sum_{r \in D} \lambda_{r}(x) \Lambda_{r}:=\sum_{j=0}^{\infty} \sum_{r \in D_{j}} \lambda_{r}(x) \Lambda_{r},
$$

with uniform convergence on $[0,1]$. The Schauder scalar coefficients $\lambda_{r}(x)$ are given by

$$
\lambda_{r}(x)=x(r)-\frac{x\left(r^{+}\right)+x\left(r^{-}\right)}{2}, \quad r \in D_{j}, j \geq 1,
$$

and in the special case $j=0$ by

$$
\lambda_{0}(x)=x(0), \quad \lambda_{1}(x)=x(1) .
$$

By Ciesielski (1980), $\left\{\Lambda_{r} ; r \in D\right\}$ is a Schauder basis of $H_{\alpha}^{0}[0,1]$ and we have the equivalence of norms

$$
\|x\|_{\alpha} \sim\|x\|_{\alpha}^{\text {seq }}:=\sup _{j \geq 0} 2^{j \alpha} \max _{r \in D_{j}}\left|\lambda_{r}(x)\right| .
$$

This equivalence enables us to exploit the following tightness criterion.

Theorem 3. The sequence $\left(\zeta_{n}\right)$ of random elements in the Hölder space $H_{\alpha}^{0}[0,1]$ is tight if and only if the following two conditions are satisfied:

(i) $\lim _{b \rightarrow \infty} \sup _{n} \mathrm{P}\left\{\left|\zeta_{n}(0)\right|>b\right\}=0$; 
(ii) for each $\varepsilon>0$

$$
\lim _{J \rightarrow \infty} \limsup _{n \rightarrow \infty} \mathrm{P}\left\{\sup _{i \geq J} 2^{j \alpha} \max _{r \in D_{j}}\left|\lambda_{r}\left(\zeta_{n}\right)\right|>\varepsilon\right\}=0 .
$$

For the proof see [8] (where condition (ii) is written with $\sup _{n \geq 1}$ instead of $\lim \sup _{n \rightarrow \infty}$, but the reduction to the lim sup is classical).

\section{Proof of Theorem 1}

Necessity. By the continuous mapping theorem, the convergence in distribution of $n^{-1 / 2} \xi_{n}$ yields, for each $\delta>0$,

$$
\omega_{\alpha}\left(n^{-1 / 2} \xi_{n}, \delta\right) \stackrel{\mathcal{D}}{\longrightarrow} \omega_{\alpha}(W, \delta) .
$$

Since the supremum giving the Hölder norm of a polygonal line is reached at two vertices,

$$
\omega_{\alpha}\left(n^{-1 / 2} \xi_{n}, \delta\right)=n^{-1 / 2+\alpha} \max _{|k-j| \leq n \delta} \frac{\left|S_{k}-S_{j}\right|}{|k-j|^{\alpha}} .
$$

For $n$ such that $1 / n \leq \delta$ we have

$$
\begin{aligned}
\mathrm{P}\left(n^{-1 / 2+\alpha} \max _{1 \leq k \leq n}\left|X_{k}\right|>t\right) & =\mathrm{P}\left(n^{-1 / 2+\alpha} \max _{0<|k-j| \leq 1} \frac{\left|S_{k}-S_{j}\right|}{|k-j|^{\alpha}}>t\right) \\
& \leq \mathrm{P}\left(n^{-1 / 2+\alpha} \max _{0<|k-j| \leq n \delta} \frac{\left|S_{k}-S_{j}\right|}{|k-j|^{\alpha}}>t\right) \\
& =\mathrm{P}\left(\omega_{\alpha}\left(n^{-1 / 2} \xi_{n}, \delta\right)>t\right) .
\end{aligned}
$$

By the convergence (5)

$$
\limsup _{n \rightarrow \infty} \mathrm{P}\left(\omega_{\alpha}\left(n^{-1 / 2} \xi_{n}, \delta\right)>t\right) \leq \mathrm{P}\left(\omega_{\alpha}(W, \delta)>t\right) .
$$

This yields for each $\delta>0$

$$
\limsup _{n \rightarrow \infty} \mathrm{P}\left(n^{-1 / 2+\alpha} \max _{1 \leq k \leq n}\left|X_{k}\right|>t\right) \leq \mathrm{P}\left(\omega_{\alpha}(W, \delta)>t\right) .
$$

Since $\mathrm{P}\left(\omega_{\alpha}(W, \delta)>t\right) \rightarrow 0$ as $\delta \rightarrow 0$, we easily conclude the necessity of condition (2).

Sufficiency. The convergence of the finite-dimensional distributions follows by the Donsker-Prokhorov invariance principle, since condition (2) yields $\mathrm{E} X_{1}^{2}<\infty$. In what follows we assume that $\mathrm{E} X_{1}^{2}=1$.

To prove tightness we shall use Theorem 3 . Throughout

$$
r_{k}=r_{k j}=k 2^{-j}, \quad k=0,1, \ldots, 2^{j}, j=1,2, \ldots .
$$

Since $\xi_{n}(0)=0$, we have to check the second condition of Theorem 3 only. To this aim we have to estimate

$$
P=\mathrm{P}\left(\sup _{J \leq j} 2^{\alpha j} n^{-1 / 2} \max _{1 \leq k<2^{j}}\left|\xi_{n}\left(r_{k+1}\right)-\xi_{n}\left(r_{k}\right)\right| \geq \varepsilon\right) \leq P_{1}(J, n, \varepsilon)+P_{2}(n, \varepsilon),
$$

where

$$
P_{1}(J, n, \varepsilon)=\mathrm{P}\left(\sup _{J \leq j \leq \log n} 2^{\alpha j} n^{-1 / 2} \max _{1 \leq k \leq 2^{j}}\left|\xi_{n}\left(r_{k+1}\right)-\xi_{n}\left(r_{k}\right)\right| \geq \varepsilon\right)
$$

and

$$
P_{2}(n, \varepsilon)=\mathrm{P}\left(\sup _{j>\log n} 2^{\alpha j} n^{-1 / 2} \max _{1 \leq k \leq 2^{j}}\left|\xi_{n}\left(r_{k+1}\right)-\xi_{n}\left(r_{k}\right)\right| \geq \varepsilon\right) .
$$


Here and throughout the paper, log denotes the logarithm with basis 2 , while $\ln$ denotes the natural $\operatorname{logarithm}\left(\log \left(2^{x}\right)=x=\ln \left(\mathrm{e}^{x}\right)\right)$.

Estimation of $P_{2}(n, \varepsilon)$. If $j>\log n$, then

$$
r_{k+1}-r_{k}=(k+1) 2^{-j}-k 2^{-j}=2^{-j}<1 / n
$$

and therefore if $r_{k} \in[v / n,(v+1) / n)$, then either

$$
r_{k+1} \in[v / n,(v+1) / n)
$$

or

$$
r_{k+1} \in[(v+1) / n,(v+2) / n),
$$

where $1 \leq v \leq n-2$ depends on $k$ and $j$. If both $r_{k}$ and $r_{k+1}$ are in the same interval, then we have

$$
\left|\xi_{n}\left(r_{k+1}\right)-\xi_{n}\left(r_{k}\right)\right|=\left|X_{v+1}\right| 2^{-j} n \leq 2^{-j} n \max _{1 \leq v \leq n}\left|X_{v}\right| .
$$

If $r_{k}$ and $r_{k+1}$ are in different intervals, then

$$
\begin{aligned}
\left|\xi_{n}\left(r_{k+1}\right)-\xi_{n}\left(r_{k}\right)\right| & \leq\left|\xi_{n}\left(r_{k}\right)-\xi_{n}((v+1) / n)\right|+\left|\xi_{n}((v+1) / n)-\xi_{n}\left(r_{k+1}\right)\right| \\
& \leq 2^{-j+1} n \max _{1 \leq v \leq n}\left|X_{v}\right| .
\end{aligned}
$$

With both cases taken into account we obtain

$$
\begin{aligned}
P_{2}(n, \varepsilon) & \leq \mathrm{P}\left(\max _{j>\log n} 2^{\alpha j} n 2^{-j} n^{-1 / 2} \max _{1 \leq v \leq n}\left|X_{v}\right| \geq \varepsilon / 2\right) \\
& \leq \mathrm{P}\left(n^{\alpha-1 / 2} \max _{1 \leq v \leq n}\left|X_{v}\right| \geq \varepsilon / 2\right) \\
& \leq n \mathrm{P}\left(\left|X_{1}\right| \geq(\varepsilon / 2) n^{1 / p}\right) .
\end{aligned}
$$

Hence, due to condition (2), for each $\varepsilon>0$

$$
\lim _{n \rightarrow \infty} P_{2}(n, \varepsilon)=0 .
$$

Estimation of $P_{1}(J, n, \varepsilon)$. Let $u_{k}=\left[n r_{k}\right]$. Then

$$
u_{k} \leq n r_{k} \leq u_{k}+1
$$

and

Therefore

$$
u_{k}+1 \leq u_{k+1} \leq n r_{k+1} \leq u_{k+1}+1 .
$$

$$
\left|\xi_{n}\left(r_{k+1}\right)-\xi_{n}\left(r_{k}\right)\right| \leq\left|\xi_{n}\left(r_{k+1}\right)-S_{u_{k+1}}\right|+\left|S_{u_{k+1}}-S_{u_{k}}\right|+\left|S_{u_{k}}-\xi_{n}\left(r_{k}\right)\right| .
$$

Since $\left|S_{u_{k}}-\xi_{n}\left(r_{k}\right)\right| \leq\left|X_{u_{k}+1}\right|$ and $\left|\xi_{n}\left(r_{k+1}\right)-S_{u_{k+1}}\right| \leq\left|X_{u_{k+1}+1}\right|$, we obtain

$$
P_{1}(J, n, \varepsilon) \leq P_{1,1}(J, n, \varepsilon)+P_{1,2}(J, n, \varepsilon),
$$

where

$$
\begin{aligned}
P_{1,1}(J, n, \varepsilon):=\mathrm{P} & \left(\sup _{J \leq j \leq \log n} 2^{\alpha j} n^{-1 / 2} \max _{1 \leq k \leq 2^{j}}\left|S_{u_{k+1}}-S_{u_{k}}\right| \geq \varepsilon / 3\right) \\
P_{1,2}(J, n, \varepsilon) & :=\mathrm{P}\left(\max _{J \leq j \leq \log n} 2^{\alpha j} n^{-1 / 2} \max _{1 \leq v \leq n}\left|X_{v}\right| \geq \varepsilon / 6\right) \\
& \leq \mathrm{P}\left(n^{\alpha-1 / 2} \max _{1 \leq v \leq n}\left|X_{v}\right| \geq \varepsilon / 6\right) \\
& \leq n \mathrm{P}\left(\left|X_{1}\right|>n^{1 / p} \varepsilon / 6\right) .
\end{aligned}
$$


Since $P_{1,2}(J, n, \varepsilon) \rightarrow 0$ as $n \rightarrow \infty$ due to condition (2), we have to deal with $P_{1,1}(J, n, \varepsilon)$ only. To this aim let $\delta>0$ be an arbitrary number and define the truncated random variables

$$
\widetilde{X}_{k}=X_{k} \mathbb{1}\left\{\left|X_{k}\right| \leq n^{1 / p} \delta\right\}, \quad X_{k}^{\prime}=X_{k}-\tilde{X}_{k}, \quad k=1, \ldots, n .
$$

Then

$$
P_{1,1}(J, n, \varepsilon) \leq n \mathrm{P}\left(\left|X_{1}\right| \geq \delta n^{1 / p}\right)+P_{1,1}^{\prime}(J, n, \varepsilon),
$$

where

$$
P_{1,1}^{\prime}(J, n, \varepsilon):=\mathrm{P}\left(\sup _{J \leq j \leq \log n} 2^{\alpha j} n^{-1 / 2} \max _{1 \leq k \leq 2^{j}}\left|\sum_{i=u_{k}+1}^{u_{k+1}} \widetilde{X}_{i}\right| \geq \frac{\varepsilon}{3}\right) .
$$

Since

$$
\begin{aligned}
\mathrm{E} \widetilde{X}_{i} & =\mathrm{E} X_{i} \mathbb{1}\left\{\left|X_{i}\right| \geq \delta n^{1 / p}\right\}=\int_{\delta n^{1 / p}}^{\infty} \mathrm{P}\left(\left|X_{1}\right| \geq t\right) d t \\
& \leq \sup _{t} t^{p} \mathrm{P}\left(\left|X_{1}\right| \geq t\right) \int_{\delta n^{1 / p}}^{\infty} t^{-p} d t \\
& \leq c \delta^{-p+1} n^{-1+1 / p}
\end{aligned}
$$

we have

$$
\begin{aligned}
\sup _{J \leq j \leq \log n} 2^{\alpha j} n^{-1 / 2} \max _{1 \leq k \leq 2 j}\left|\sum_{i=u_{k}+1}^{u_{k+1}} \mathrm{E} \widetilde{X}_{i}\right| & \leq c \delta^{-p+1} \sup _{J \leq j \leq \log n} 2^{\alpha j-j} n^{1 / 2} n^{-1+1 / p} \\
& \leq c \delta^{-p+1} 2^{-J}<\varepsilon / 6
\end{aligned}
$$

for large $J$. Therefore, for $n$ and $J$ large,

$$
\begin{aligned}
P_{1,1}^{\prime}(J, n, \varepsilon) & \leq \mathrm{P}\left(\sup _{J \leq j \leq \log n} 2^{\alpha j} n^{-1 / 2} \max _{1 \leq k \leq 2^{j}}\left|\sum_{i=u_{k}+1}^{u_{k+1}}\left(\widetilde{X}_{i}-\mathrm{E} \widetilde{X}_{i}\right)\right| \geq \varepsilon / 6\right) \\
& \leq(\varepsilon / 6)^{-q} \sum_{j=J}^{\log n} 2^{q \alpha j} n^{-q / 2} 2^{j} \mathrm{E}\left|\sum_{i=u_{k}+1}^{u_{k+1}}\left(\widetilde{X}_{i}-\mathrm{E} \widetilde{X}_{i}\right)\right|^{q},
\end{aligned}
$$

where $q>2$. By Rosenthal's inequality (accounting $\mathrm{E} X_{1}^{2}=1$ )

$$
\begin{aligned}
\mathrm{E}\left|\sum_{i=u_{k}+1}^{u_{k+1}}\left(\widetilde{X}_{i}-\mathrm{E} \widetilde{X}_{i}\right)\right|^{q} & \leq c\left[\left(u_{k+1}-u_{k}\right)^{q / 2}\left(\mathrm{E} X_{1}^{2}\right)^{q / 2}+\left(u_{k+1}-u_{k}\right) \mathrm{E}\left|\widetilde{X}_{1}\right|^{q}\right] \\
& \leq c\left[\left(n 2^{-j}\right)^{q / 2}+n 2^{-j} \delta^{q-p} n^{(q-p) / p}\right],
\end{aligned}
$$

since

$$
\mathrm{E}\left|\widetilde{X}_{1}\right|^{q} \leq \int_{0}^{\delta n^{1 / p}} u^{q-1} \mathrm{P}\left(\left|X_{1}\right|>u\right) d u \leq n^{(q-p) / p} \delta^{q-p} \sup _{u} u^{p} \mathrm{P}\left(\left|X_{1}\right|>u\right) .
$$

Substituting this estimate we obtain (denoting $c_{0}=c(\varepsilon / 6)^{-q}$ )

$$
\begin{aligned}
P_{1,1}^{\prime}(J, n, \varepsilon) & \leq c_{0} \sum_{j=J}^{\log n} 2^{q \alpha j} n^{-q / 2} 2^{j}\left[\left(n 2^{-j}\right)^{q / 2}+n 2^{-j} \delta^{q-p} n^{(q-p) / p}\right] \\
& \leq c_{0} \sum_{j=J}^{\log n} 2^{q \alpha j} n^{-q / 2} 2^{j}\left(n 2^{-j}\right)^{q / 2}+c_{0} \delta^{q-p} \sum_{j=J}^{\log n} 2^{q \alpha j} n^{-q / 2} 2^{j} n 2^{-j} n^{(q-p) / p} \\
& \leq c_{0} \sum_{j=J}^{\log n} 2^{(q \alpha+1-q / 2) j}+c_{0} \delta^{q-p} n^{-q \alpha} \sum_{j=J}^{\log n} 2^{q \alpha j} .
\end{aligned}
$$


Choosing

we obtain

$$
q>\left(\frac{1}{2}-\alpha\right)^{-1}
$$

$$
P_{1,1}^{\prime}(J, n, \varepsilon) \leq c_{0} 2^{-(q(1 / 2-\alpha)-1) J}+c_{0} c \delta^{q-p}<\delta
$$

for big $J$. The result is proved.

Proof of Corollary 2. Sufficiency follows by Theorem 1 and the continuous mapping theorem. Necessity follows from the proof of Theorem 1.

\section{Distribution of Some Brownian Hölder norms}

Let us recall the following well-known representation of Brownian motion which goes back to Lévy and Kampé de Fériet and was generalized by Shepp (1966) with any orthonormal basis.

Theorem 4. Let $\left\{X_{r} ; r \in D^{*}\right\}$ be a sequence of independent standard normal random variables. Then the series

$$
W(t):=\sum_{r \in D^{*}} X_{r} \int_{0}^{t} H_{r}(s) d s, \quad t \in[0,1],
$$

converges a.s. uniformly on $[0,1]$ and $W$ is a Brownian motion started at 0 . Removing the first term in (6) gives a Brownian bridge

$$
B(t):=W(t)-t W(1)=\sum_{j=1}^{\infty} \sum_{r \in D_{j}} X_{r} \int_{0}^{t} H_{r}(s) d s, \quad t \in[0,1] .
$$

In view of (2) and (4), the Schauder coefficients of $W$ are

$$
\lambda_{1}(W)=X_{1}, \quad \lambda_{r}(W)=2^{-(j+1) / 2} X_{r}, \quad r \in D_{j}, j \geq 1 .
$$

The Hölder sequential norms of $W$ and $B$ may now be written as

$$
\|B\|_{\alpha}^{\text {seq }}=\frac{1}{\sqrt{2}} \sup _{j \geq 1} 2^{j(\alpha-1 / 2)} \max _{r \in D_{j}}\left|X_{r}\right|, \quad\|W\|_{\alpha}^{\text {seq }}=\max \left\{\left|X_{1}\right|,\|B\|_{\alpha}^{\text {seq }}\right\} .
$$

The distribution function of $\|B\|_{\alpha}^{\text {seq }}$ may be conveniently expressed in terms of the error function:

$$
\operatorname{erf} x=\frac{2}{\sqrt{\pi}} \int_{0}^{x} \exp \left(-s^{2}\right) d s=\mathrm{P}\left(\left|X_{1}\right| \leq x \sqrt{2}\right), \quad x \geq 0 .
$$

The following asymptotic expansion will be useful:

$$
\operatorname{erf} x=1-\frac{1}{x \sqrt{\pi}} \exp \left(-x^{2}\right)\left(1+O\left(x^{-2}\right)\right), \quad x \rightarrow \infty .
$$

Theorem 5. If $\alpha<\frac{1}{2}$, then $\|B\|_{\alpha}^{\text {seq }}$ and $\|W\|_{\alpha}^{\text {seq }}$ are almost surely finite and their distribution functions are given by

$$
\mathrm{P}\left(\|B\|_{\alpha}^{\text {seq }} \leq x\right)=\prod_{j=1}^{\infty}\left\{\operatorname{erf}\left(2^{j(1 / 2-\alpha)} x\right)\right\}^{2^{j-1}}, \quad x>0,
$$

and

$$
\mathrm{P}\left(\|W\|_{\alpha}^{\text {seq }} \leq x\right)=\operatorname{erf}\left(2^{-1 / 2} x\right) \prod_{j=1}^{\infty}\left\{\operatorname{erf}\left(2^{j(1 / 2-\alpha)} x\right)\right\}^{2^{j-1}}, \quad x>0 .
$$

The support of these distributions is $[0, \infty)$. 
Proof. In view of (8), consider the nondecreasing sequence of random variables $\left(M_{J}\right)_{J \geq 1}$, where

$$
M_{J}:=\frac{1}{\sqrt{2}} \max _{1 \leq j \leq J} 2^{j(\alpha-1 / 2)} \max _{r \in D_{j}}\left|X_{r}\right| .
$$

Regarding $\|B\|_{\alpha}^{\text {seq }}$ as a random element in $[0, \infty]$, we have, for each $x \in \mathbf{R}$,

$$
\mathrm{P}\left(M_{J} \leq x\right) \downarrow \mathrm{P}\left(\|B\|_{\alpha}^{\text {seq }} \leq x\right)=: F_{B, \alpha}(x) \quad \text { as } J \rightarrow \infty .
$$

So to prove the theorem it is enough to show that

a) $F_{B, \alpha}(x)>0$ for each $x \in[0, \infty)$, and

b) $\lim _{x \rightarrow \infty} F_{B, \alpha}(x)=1$.

Now by independence and equal distribution of the $X_{r}$ 's

$$
\mathrm{P}\left(M_{J} \leq x\right)=\prod_{j=1}^{J} \operatorname{erf}\left(2^{j(1 / 2-\alpha)} x\right)^{2^{j-1}}
$$

Taking logarithms in (12) and using (9), it is easily seen that a) follows from the pointwise convergence on $(0, \infty)$ of the series $\sum_{j=1}^{\infty} u_{j}(x)$, where

$$
u_{j}(x)=\frac{1}{x} 2^{j(1 / 2+\alpha)} \exp \left(-x^{2} 2^{j(1-2 \alpha)}\right) .
$$

Then for each $x \in(0, \infty)$ the function $F_{B, \alpha}(x)$ is represented by the convergent infinite product (10), and we obtain b) applying the bounded convergence theorem (with $x \rightarrow \infty$ ) to the series

$$
\ln F_{B, \alpha}(x)=\sum_{j=1}^{\infty} 2^{j-1} \ln \operatorname{erf}\left(2^{j(1 / 2-\alpha)} x\right) .
$$

\section{A statistical application}

As an application of the Hölderian invariance principle, consider the problem of testing a change in the mean under the so-called epidemic alternative (see [2] and the references therein). The problem may be described as follows. Let $X_{1}, X_{2}, \ldots, X_{n}$ be independent random variables. We want to test the null hypothesis of constant mean,

$\left(H_{0}\right): X_{1}, \ldots, X_{n}$ are identically distributed with mean $\mathrm{E} X_{1}=0$ and variance

$$
\operatorname{Var}\left(X_{1}\right)=\sigma^{2}>0
$$

against the epidemic alternative

$\left(H_{A}\right)$ : there are integers $1<k^{*}<m^{*}<n$ and a constant $c \neq 0$ such that

$$
\mathrm{E} X_{i}=c \mathbb{1}_{\left(k^{*}<i \leq m^{*}\right)}, \quad i=1,2, \ldots, n .
$$

We suggest the following test statistics:

$$
\mathrm{UI}(n, \alpha)=n^{\alpha} \max _{1 \leq i<j \leq n} \frac{|S(j)-S(i)-S(n)(j-i) / n|}{[(j-i)(1-(j-i) / n)]^{\alpha}}
$$

and

$$
\mathrm{DI}(n, \alpha)=\max _{1 \leq j \leq \log n} 2^{j \alpha} \max _{r \in D_{j}}\left|S(n r)-\frac{1}{2}\left(S\left(n r^{+}\right)+S\left(n r^{-}\right)\right)\right| .
$$

Introduce the following random variables:

$$
\mathrm{UI}(\alpha)=\sup _{0<|t-s|<1} \frac{|B(s)-B(t)|}{(s-t)^{\alpha}(1-(t-s))^{\alpha}}
$$


and

$$
\mathrm{DI}(\alpha)=\sup _{j \geq 1} 2^{j \alpha} \max _{r \in D_{j}}\left|W(r)-\frac{1}{2}\left(W\left(r^{+}\right)+W\left(r^{-}\right)\right)\right| .
$$

Theorem 6. Let $p=1 /\left(\frac{1}{2}-\alpha\right)$. If under $H_{0}$

$$
\lim _{t \rightarrow \infty} t^{p} \mathrm{P}\left(\left|X_{1}\right|>t\right)=0,
$$

then

$$
\sigma^{-1} n^{-1 / 2} \mathrm{UI}(n, \alpha) \stackrel{\mathcal{D}}{\longrightarrow} \mathrm{UI}(\alpha)
$$

and

$$
\sigma^{-1} n^{-1 / 2} \mathrm{DI}(n, \alpha) \stackrel{\mathcal{D}}{\longrightarrow} \mathrm{DI}(\alpha) .
$$

Proof. Without loss of generality, we assume that $\sigma=1$. An elementary computation gives

where

$$
n^{-1 / 2} \mathrm{UI}(n, \alpha)=\max _{1 \leq i<j \leq n} I\left(n^{-1 / 2} \xi_{n}, i / n, j / n\right)
$$

$$
I(x, s, t)=\frac{|x(t)-x(s)-(t-s) x(1)|}{(t-s)^{\alpha}(1-(t-s))^{\alpha}} .
$$

Let $N_{\alpha}:=\left\{x \in H_{\alpha}^{o}[0,1] ; x(0)=0\right\}$ and consider the functionals $L_{n}, L$ defined on this closed subspace $N_{\alpha}$ by

$$
L_{n}(x)=\max _{1 \leq i<j \leq n} I(x, i / n, j / n), \quad L(x)=\sup _{0<s<t<1} I(x, s, t) .
$$

For any $x \in N_{\alpha},(s, t) \mapsto I(x, s, t)$ can be extended to a continuous function on the compact set $\{0 \leq s \leq t \leq 1\}$, and then $L_{n}(x)$ clearly converges to $L(x)$.

By Lemma 7 below, the sequence $\left(L_{n}\right)$ is equicontinuous and by a well-known corollary of Ascoli's theorem converges uniformly to $L$ on every compact set of $N_{\alpha}$. This fact combined with the tightness of $\left(n^{-1 / 2} \xi_{n}\right)$ in $N_{\alpha}$ (which follows from our hypothesis (13) and Theorem 1) easily gives the convergence in probability to zero of $L\left(n^{-1 / 2} \xi_{n}\right)-$ $L_{n}\left(n^{-1 / 2} \xi_{n}\right)$. So we have

$$
n^{-1 / 2} \mathrm{UI}(n, \alpha)=L\left(n^{-1 / 2} \xi_{n}\right)+o_{P}(1) \quad \text { as } n \rightarrow \infty .
$$

Now (14) follows from Theorem 1 by a continuous mapping.

For the proof of $(15)$, since $S(n t)=\xi_{n}(t)-(n t-[n t]) X_{[n t]+1}$, we have for $r \in D$

$$
S(n r)-\frac{1}{2}\left(S\left(n r^{+}\right)+S\left(n r^{-}\right)\right)=\lambda_{r}\left(\xi_{n}\right)+Z(n, r)
$$

for a random variable $Z(n, r)$ such that $|Z(n, r)| \leq 2 \max _{k \leq n}\left|X_{k}\right|$. It follows that

$$
n^{-1 / 2} \mathrm{DI}(n, \alpha)=\left\|E_{n}\left(n^{-1 / 2} \xi_{n}\right)\right\|_{\alpha}^{\text {seq }}+n^{\alpha-1 / 2} Z_{n},
$$

where $Z_{n}$ is a nonnegative random variable such that $0 \leq Z_{n} \leq 2 \max _{k \leq n}\left|X_{k}\right|$, and $E_{n}$ is the projection onto the subspace generated by the triangular functions

$$
\left(\Lambda_{r}, r \in D_{j}, j \leq \log n\right) .
$$

The $E_{n}$ are continuous, and for every $x \in H_{\alpha}^{o}[0,1],\left\|E_{n}(x)\right\|_{\alpha}^{\text {seq }}$ is nondecreasing (in $n$ ) and converges to $\|x\|_{\alpha}^{\text {seq }}$. It follows that this convergence is uniform over every compact set of $N_{\alpha}$ (e.g., by Dini's theorem), so that by tightness of $\left(n^{-1 / 2} \xi_{n}\right)$,

$$
\left\|E_{n}\left(n^{-1 / 2} \xi_{n}\right)\right\|_{\alpha}^{\text {seq }}=\left\|n^{-1 / 2} \xi_{n}\right\|_{\alpha}^{\text {seq }}+o_{P}(1) \text {. }
$$


Since condition (13) gives also that $n^{\alpha-1 / 2} \max _{k \leq n}\left|X_{k}\right|$ goes to zero in probability, we obtain (15) from Theorem 1 by a continuous mapping.

Lemma 7. The functionals $L_{n}$ defined by (16) satisfy on $N_{\alpha}$ the Lipschitz condition,

$$
\left|L_{n}(x+y)-L_{n}(x)\right| \leq 2^{2+\alpha}\|y\|_{\alpha}, \quad n \geq 2 .
$$

The functional $L$ satisfies the same inequality.

Proof. For simplicity we write $f(u):=u^{\alpha}(1-u)^{\alpha}, u \in[0,1]$. Putting $L_{\infty}:=L$, we agree to include the case $n=\infty$ in each condition like, say, $n \geq 2$.

Obviously $I(x, s, t)$ is subadditive in $x$, and for $n \geq 2$, the $L_{n}$ inherit this property:

$$
L_{n}(x+y) \leq L_{n}(x)+L_{n}(y), \quad x, y \in N_{\alpha} .
$$

Clearly these functionals are homogeneous: $L_{n}(c x)=|c| L_{n}(x)$. This together with (17) gives

$$
\left|L_{n}(x+y)-L_{n}(x)\right| \leq L_{n}(y), \quad x, y \in N_{\alpha} .
$$

So it only remains to check that $L_{n}(y) \leq 2^{2+\alpha}\|y\|_{\alpha}$, which in turn reduces to

$$
I(y, s, t) \leq 2^{2+\alpha}\|y\|_{\alpha}
$$

If $0<t-s \leq \frac{1}{2}, f(t-s) \geq(t-s)^{\alpha} 2^{-\alpha}$, so

$$
I(y, s, t) \leq 2^{\alpha} \frac{|y(t)-y(s)|}{(t-s)^{\alpha}}+2^{\alpha}(t-s)^{1-\alpha}|y(1)| \leq 2^{1+\alpha}\|y\|_{\alpha} .
$$

If $\frac{1}{2}<t-s \leq 1$, we have $(1-t)+s \leq \frac{1}{2}$, and as $f(u)=f(1-u), f(t-s)$ is bigger than $f(1-t)$ and $f(s)$. It follows that

$$
I(y, s, t) \leq \frac{|y(t)-y(1)|}{f(1-t)}+\frac{(1-t)|y(1)|}{f(1-t)}+\frac{|y(s)|}{f(s)}+\frac{s|y(1)|}{f(s)} \leq 2^{2+\alpha}\|y\|_{\alpha} .
$$

The proof is complete.

\section{BIBLIOGRAPHY}

1. Z. Ciesielski, On the isomorphisms of the spaces $H_{\alpha}$ and $m$, Bull. Acad. Pol. Sci. Ser. Sci. Math. Phys. 8 (1960), 217-222. MR 24:A2234

2. M. Csörgö and L. Horváth, Limit Theorems in Change-Point Analysis, John Wiley \& Sons, New York, 1997.

3. R. V. Erickson, Lipschitz smoothness and convergence with applications to the central limit theorem for summation processes, Ann. Probab. 9 (1981), 831-851. MR 83h:60006

4. D. Hamadouche, Invariance Principles in Hölder Spaces, Portugaliae Mathematica 57 (2000), 127-151. MR 2001g:60075

5. B. S. Kashin and A. A. Saakyan, Orthogonal Series, Translations of Mathematical Monographs, vol. 75, AMS, Providence, Rhode Island, 1989. MR 90g:42001

6. G. Kerkyacharian and B. Roynette, Une démonstration simple des théorèmes de Kolmogorov, Donsker et Itô-Nisio, C. R. Acad. Sci. Paris Série I 312 (1991), 877-882. MR 92g:60009

7. J. Lamperti, On convergence of stochastic processes, Trans. Amer. Math. Soc. 104 (1962), 430-435. MR 26:804

8. A. Račkauskas and Ch. Suquet, Central limit theorem in Hölder spaces, Probab. Math. Stat. 19 (1999), 155-174. MR 2000k:60012 
9. - On the Hölderian functional central limit theorem for i.i.d. random elements in Banach space, Proceedings of the Fourth Hungarian Colloquium on Limit Theorems of Probability and Statistics (1999), Pub. IRMA Lille 50-III, 1999. MR 2004a:60004

10. L. A. Shepp, Radon-Nikodym derivatives of Gaussian measures, Annals of Mathematical Statistics 37 (1966), 321-354. MR 32:8408

Vilnius University, Department of Mathematics, Naugarduko 24, Lt-2006 Vilnius, Lithuania

E-mail address: Alfredas.Rackauskas@maf.vu.lt

Université des Sciences et Technologies de Lille, Laboratoire de Mathématiques ApPliquées F.R.E. CNRS 2222, BÂt. M2, U.F.R. De Mathématiques, F-59655 Villeneuve D’AscQ Cedex France

E-mail address: Charles.Suquet@univ-Lille1.fr

Received 4/APR/2002

Originally published in English 